•研究报告・

\title{
中国公众的国际野生动物保护意愿调查：以非洲象 为例
}

张馨予, 胡宇轩, 张忠义, 傅铨涵, 谢屹* 北京林业大学经济管理学院, 北京 100083

摘要：我国高度重视野生动物保护事业, 认真履行野生动物保护国际义务, 积极鼓励公众参与, 以扩大野生动物保护事业的 公众基础。已有文献多关注了公众的国内野生动物保护意愿，鲜有文献关注公众对国际野生动物的保护意愿，难以为促进公 众参与国际野生动物保护事业提供决策参考。本研究以全球旗舰物种非洲象 (Loxodonta africana)为例, 结合非洲象保护的相 关研究与实践, 构建拓展的计划行为理论框架, 通过线下和线上调研获取数据, 运用结构方程模型, 从态度、规范、知觉行为 控制、过去经验及个体特征五个方面，分析了我国公众的非洲象保护意愿及影响因素。结果表明: (1) 68.5\%的公众具有非洲 象保护愿意; (2)公众规范(系数为0.422)、过去经验(系数为 0.253 )、知觉行为控制(系数为 0.160 )、保护态度(系数为 0.156 )对保 护意愿存在显著的正向影响; 男性公众(系数为 -0.054 )的保护意愿低于女性公众; 居住在西部地区的公众(系数为0.066)保护 意愿更高; (3)模型整体通过了拟合检验, 表明研究结果具有稳健性。本研究的政策建议如下: (1)明确政策导向作用, 提升公众 的道德义务感和社会责任感; (2)加强宣传教育, 丰富公众知识经验, 培育公众积极的保护态度; (3)拓宽保护参与渠道, 提高 公众知觉行为控制; (4)制定合理方案，提升保护宣教等实践活动成效。

关键词：非洲象; 保护意愿; 计划行为理论; 国际野生动物保护; 公众

张馨予, 胡宇轩, 张忠义, 傅铎涵, 谢屹 (2021) 中国公众的国际野生动物保护意愿调查: 以非洲象为例. 生物多样性, 29, 1358-1368. doi: 10.17520/biods.2021082.

Zhang XY, Hu YX, Zhang ZY, Fu YH, Xie Y (2021) Chinese public willingness of international wildlife conservation: A case study of African elephant. Biodiversity Science, 29, 1358-1368. doi: 10.17520/biods.2021082.

\section{Chinese public willingness of international wildlife conservation: A case study of African elephant}

\author{
Xinyu Zhang ${ }^{(D)}$, Yuxuan Hu, Zhongyi Zhang, Yuhan Fu, Yi Xie* \\ School of Economics and Management, Beijing Forestry University, Beijing 100083
}

\begin{abstract}
Aim: The government of China has been attaching great importance to wildlife conservation, actively performing international obligations of global wildlife protection and vigorously promoting public participation to expand public foundation of the conservation. Although the public intention to defend domestic wild species has been presented in literatures, their willingness to conserve global wildlife remains uncommon in the relevant literatures, which provide little decision-making references to promote public participation in global wildlife conservation. We therefore studied Chinese public willingness of nondomestic species conservation and its influencing factors.

Method: The global flagship species African elephant (Loxodonta Africana) was selected as an invested subject and relevant studies and practices of their conservation were reviewed to construct the extended framework of the theory of planned behavior. Data were collected on- or off-line for analyses with structural equation modeling.

Results: The results indicated that $68.5 \%$ of the public responded their positive willingness of African elephant conservation. The factors of individual norm [coefficient (hereinafter coef.) 0.422], past experience (coef. 0.253), perceived behavioral control (coef. 0.160), and attitude (coef. 0.156) illustrated positive impacts on the public
\end{abstract}

收稿日期: 2021-03-07; 接受日期: 2021-06-30

基金项目：国家自然科学基金(718611470001)和国家林业和草原局林业软科学项目(2019131025)

* 通讯作者 Author for correspondence. E-mail: yixie@bjfu.edu.cn 
willingness of African elephant conservation with a successively increasing impacting degree. The results also showed that male respondents (coef.-0.054) had lower conservatio $n$ willingness, while the interviewees living in the western regions (coef. 0.066) possessed a higher willingness. The model passed the statistical fitting testification, confirming the reliability of the results.

Suggestion: We therefore suggests that it is necessary to explicitly stipulate the role of policy guidance and enhance the senses of moral obligation and social responsibility among the public. It also proposes to strengthen publicity and education in the public to enrich their knowledge and experience and to cultivate their positive attitude towards conservation, and to broaden the channels of public conservation participation and improve their perception and behavior control. The recommendation finally includes to formulate reasonable programs and elevate the effectiveness of practical activities such as conservation publicity and education.

Key words: African elephant; conservation willingness; the theory of planned behavior; global wildlife conservation; public

随着全球政治经济的一体化, 野生动物保护国 际合作得到世界各国的重视, 成为东盟峰会、中非 论坛、二十国集团领导人峰会、《生物多样性公约》 缔约方大会等国际外交活动中的一个重要议题 (Phang et al, 2020)。与此同时, 公众个人和基层社 区、社会组织等社会公众(见《光明日报》2020-03-04 第6版)在野生动物保护中的重要性逐渐凸显, 成为 以政府为主导的野生动物保护工作的有力支持 (Halkos \& Jones, 2012)。如何进一步提升社会公众 在野生动物保护工作中的积极作用, 受到管理层和 学术界的共同关注。

公众个人(下文简称公众)在野生动物保护中具 有多重身份, 可以作为捐赠者、监督员、讲解员(梦 梦, 2013)。有关公众对本国物种保护意愿及其影响 因素的研究众多。如关于哈尔滨市民对东北虎保护 投入意愿的研究表明, 市民平均每月的支付意愿为 88.56元/(户・年) (周学红等, 2009)。公众对本国物种 的保护意愿受到经验、情感、物种特征、社会人口 特征等因素的影响。其中, 经验因素包括对保护对 象的熟悉度(Martín-López et al, 2007)、接触经验等 (Turpie, 2003); 情感因素包括对保护对象的态度 (Browne-Nuñez et al, 2013; Loyau \& Schmeller, 2017)、社会规范(Langin \& Jacobson, 2012)、管理制 度偏好(Subroy et al, 2018)、价值取向(Ojea \& Loureiro, 2007); 物种特征包括受保护对象的数量 (Bandara \& Tisdell, 2005)、是否具有标志性; 社会人 口特征包括公众的性别、年龄、收入水平等 (Dalrymple et al, 2012; Neupane et al, 2017)。然而, 上述四个方面的影响因素没有纳入现有研究统一 的分析框架，制约了结果对决策制订的参考价值。
比较而言, 公众对本国物种和非本国物种的保 护意愿可能存在差异。Haefele (2019)对比加拿大、 美国、墨西哥三国居民对同一物种的保护意愿发现, 公众更倾向于参与本国物种保护。少数关于公众对 非本国物种的保护意愿研究表明, 公众愿意为遥远 地区的物种保护提供支持: 英国居民愿意参与坦桑 尼亚濒危物种保护, 尤其针对特有物种大猩猩, 居 民支付意愿达到15.90英镑/(户・年) (Morse-Jones et al, 2012); 中国网民对非洲象保护捐赠意愿约83.62 元/(户・年) (Wang et al, 2018), 且针对不同类型保护 活动的投入意愿存在差异(Wang et al, 2020)。然而, 关于公众对非本国物种保护意愿的形成机理研究 鲜见, 难以为促进公众参与国际野生动物保护提 供决策支撑。

非洲象是全球生物多样性保护领域的旗舰物 种, 得到国际社会的高度关注(Caughley et al, 1990)。国际社会在资金、技术、装备等方面为非洲 象分布国家予以支持(袁丹丹, 2018), 以弥补这些国 家由于经济发展水平低下, 在物种保护和管理方面 的财政资源投入的不足(Kideghesho, 2016)。国际野 生动物保护投入资金呈现由富有国家和地区向欠 发达国家和地区流动的态势 (Brockington \& Scholfield, 2010)。2010-2017年, 发达国家政府及其 社会组织作为主要的援助者, 向非洲国家提供了超 过5亿美元的资金支持(Gray \& Gauntlett, 2017)。我 国政府坚持构建人类命运共同体理念, 积极参与非 洲象保护工作(秦天宝和袁昕, 2020), 为非洲象分布 国家提供了价值 1,000 万美元的物资与设备, 以加 强非洲象分布国保护能力建设(刘建周, 2020)。我国 还于2017年12月31日起全面禁止象牙商业性利用, 
以支持打击象牙及其制品非法贸易的全球行动 (Zhou et al, 2018)。然而, 非洲象仍面临的栖息地丧 失、人象冲突、非法盗猎和象牙非法贸易等威胁仍 未得到有效遏制, 种群数量持续下降(Xie, 2020), 仅 2013-2016年的减少量估计为11.7-13.5万头, 比 例约为20\% (CITES, 2019)。由于非洲象保护投入严 重短缺(EPI, 2018), 保护工作面临困境, 研究如何 增强公众参与具有十分重要的现实意义。

本研究以非洲象保护为例, 通过拓展的计划行 为理论(theory of planned behavior, TPB)建立分析框 架和提出研究假设, 结合线下和线上调研获取的样 本数据, 客观呈现公众对非洲象保护意愿及其行为 心理与其他主体特征, 采用结构方程模型验证关于 保护意愿影响因素的研究假设, 以期为提高公众对 非洲象等国际野生动物保护工作的参与积极性提 供决策参考。与现有为数不多的相关研究相比, 本 研究有两个特点: 一是首次基于社会心理学理论对 公众国际野生动物保护参与意愿开展研究, 采用的 计划行为理论适于通过行为意愿来预测没有发生 或无法观测到的行为; 二是结合国内外野生动物保 护中的公众参与实践, 对非洲象保护参与进行了较 为全面的界定, 有助于丰富现有关于保护投入意愿 (Wang et al, 2018)等单一维度的研究。

\section{理论基础和研究假设}

\section{1 理论分析框架}

计划行为理论(TPB)是通过分析行为意愿来评 价未来行为响应的成熟的社会心理学理论, 为研究 行为意愿提供了良好的、开放性的分析框架, 在公 共管理、环境保护、野生动植物保护(Miller, 2017; 史恒通等, 2019)等领域得到了广泛应用。

经典计划行为理论的核心内涵是行为意愿受 行为主体的态度、社会规范、知觉行为控制三方面 因素影响(Ajzen, 1991)。作为一个开放性的分析框 架, 不断有学者从理论和应用两方面对模型进行拓 展(Tonglet et al, 2004; 段文婷和江光荣, 2018)。在野 生动物保护领域, Sakurai (2014)提出不仅要使用理 论模型中的主观心理因素, 还要考虑经验、年龄等 其他客观因素, 以做出更准确的预测。鉴于此, 本 研究结合文献回顾及非洲象保护实践, 对经典计划 行为理论模型进行拓展, 构建分析框架(附录1), 以 探讨公众非洲象保护意愿的生成机理。
为确保分析框架得以正确应用, 需要对行为进 行明确定义(Ajzen, 2019)。本研究中的非洲象保护 活动包括改善栖息地、缓解人象冲突、反盗猎、抵 制象牙贸易四类, 均是为了应对非洲象面临的威胁; 公众参与行为指参与非洲象保护活动, 即参与上述 相关的线上活动, 诸如转发公益广告和报道、监督 和报告在线非法交易, 以及线下活动, 诸如宣传教 育、实地救助和捐助活动等。上述活动在我国野生 动物保护领域具有较广泛的实践基础(梦梦, 2013)。

\section{2 变量选择}

(1)态度。态度是对行为对象、行为本身与行为 结果是否重要的评价(Davies et al, 2002)。本研究从 三个维度测量公众的非洲象保护态度: 一是非洲象 这一保护对象是否重要, 即对于非洲象价值的评价; 二是保护非洲象是否重要, 即基于感觉与情感的经 验性评价; 三是保护结果是否重要, 即基于知识性 因素的工具性评价(Armitage \& Conner, 2001)。

(2)规范。社会规范是经典计划行为理论的一个 分析因素, 但已被证明对行为意愿的解释能力较弱, 需要进行拓展(Armitage \& Conner, 2001; Ru et al, 2019)。考虑到非洲象保护是与个体道德及社会责任 高度相关的行为, 有必要补充个体规范, 以检视个 体的内在道德规范与社会责任对行为意愿的影响。 结合规范聚焦理论 (theory of normative conduct) (Cialdini et al, 1991), 本文将社会规范和个体规范 合并为 “规范”。社会规范包括描述性规范和命令性 规范, 其中, 描述性规范指对其他重要人物是否参 与非洲象保护行为感知, 命令性规范指对其他重要 人物赞成或不赞成参与非洲象保护的感知。个体规 范指个体对参与非洲象保护这一行为持有的对错 信念(Schwartz, 1977)。

(3)知觉行为控制。知觉行为控制是个体对行为 难易程度的感知, 此方面因素的测量需要设置语义 差异或控制因子(Ajzen, 2019)。结合非洲象保护实 践, 本研究从个体的时间投入、经济投入、专业技 能投入三个维度测量公众对参与非洲象保护活动 的难易程度感知。

(4)过去经验。野生动物保护意愿很可能与过去 行为或经验有关, 如接触和参观经验、知识水平等 (Martín-López et al, 2007; Dalrymple et al, 2012)。将 过去经验纳入计划行为理论是对经典模型的有效 拓展, 能更准确地评价行为意愿和预测行为响应 
(Qi \& Ploeger, 2018; Heiny et al, 2019)。本研究中非 洲象保护的过去经验从三方面测量: 一是过去保护 行为, 指个体过去是否参与过大象及其他野生动物 保护活动的行为; 二是知识, 用于客观呈现受访者 对于非洲象生存现状及保护工作开展情况的了解 程度; 三是参与经验, 用于客观反映受访者对保护 活动参与方式与渠道的了解程度。

(5)个人特征。社会人口特征作为解释行为意愿 的背景因素, 在拓展模型中得到了广泛运用, 因此 本文加入了性别、居住地区等个人特征(Ajzen, 2011; Qi \& Ploeger, 2018; Wang et al, 2020)。

\section{3 研究假设}

鉴于上述分析，本文提出如下假设：

H1：公众非洲象保护态度正向影响其非洲象 保护意愿，即公众对非洲象保护的态度越肯定，则 保护意愿越强。

$\mathrm{H} 2$ : 公众与非洲象保护相关的规范正向影响 其非洲象保护意愿, 即公众感知到的外界压力越 大、个体道德与社会责任感越强，则保护意愿越强。

H3: 公众与非洲象保护相关的知觉行为控制 正向影响保护意愿, 即公众知觉行为控制越高, 意 味着对参与行为相关因子的控制力越强, 则保护意 愿越强。

H4: 公众过去野生动物保护经验正向影响保 护意愿, 即过去参与过相关保护活动的公众的保 护意愿更强, 且知识和参与经验越丰富保护意愿 越强。

H5: 公众个人特征显著影响保护意愿。例如, 女 性更具有同情心，保护意愿更强; 东、中、西部公众 保护意愿存在显著差异，但影响方向尚不明确。

\section{2 研究方法}

\section{1 问卷设计与数据收集}

在文献综述和群组访谈的基础上设计问卷, 以 确保问题的适用性和调研的可行性。首先, 借鉴运 用计划行为理论研究个体行为意愿的相关文献所 设计的题项(Ajzen \& Fishbein, 2008; Ajzen, 2019), 并结合非洲象保护实践, 设计了意愿、态度、规范 及知觉行为控制部分量表题项。其次, 邀请野生动 物保护专家开展焦点小组访谈, 结合对非洲象保护 的知识储备, 新开发了关于过去经验的测量题项。 最后, 于2019年开展预调研, 并据此对调查问卷进
行了修订和完善，形成正式的调查问卷(附录2)。

调查问卷包括调研目的简介、量表以及受访者 信息三部分。为了更准确地呈现公众保护意愿差异, 量表部分均采用李克特量表(Likert Scale) 7 点法 (Dawes, 2008), 即从1 (非常不同意)-7 (非常同意) 表示对测量题项的同意或不同意程度，通过受访者 的回答度量态度、规范、知觉行为控制、过去经验 和保护参与意愿。调查者的社会人口特征包括性 别、居住地区等方面信息。

本研究选取了北京、广州、福州、南京、南昌、 长沙、西安、成都、哈尔滨和沈阳 10 个城市作为调 查区域，覆盖了全国东、中、西部，其中北京、广 州、福州、南京 4 个东部城市属于过去象牙雕刻和 加工较活跃地区(Xie, 2020)。在各城市调查时, 先选 取人口流量较大但消费档次不同的超市或商场，再 根据随机抽样原则在附近定点拦截受访者, 每隔5 个人进行一次询问。调查员均为作者所在单位的研 究生，接受过专业培训。正式调研分两个阶段完成: 2020年1月，在10个城市开展实地调研获取问卷, 线下访问 1,200 次, 805 人接受访问，剔除有错误或 有遗漏信息问卷后，获得771份有效数据; 2020年2 月, 受新冠疫情影响线下调研受限, 采用网络问卷 开展补充调研以确保各调研区域均获取足够的代 表性样本, 共发放问卷230份, 回收问卷211份，获 得179份有效数据。最终本研究获得950份有效公众 样本数据，问卷有效率93.5\%。

\section{2 样本特征}

表1为样本人口统计信息。与 2010 年人口普查 数据相比, 本研究样本公众的性别分布以及东部、 中部、西部的区域分布与总体具有相似之处。样本 公众的年龄分布中，中青年群体占比较大; 教育水 平分布中, 具有本科及以上学历的样本占比高于人 口普查数据指标值。但本研究的样本与现有公众研 究的样本特征相似度高(Wang et al, 2020; 史湘莹等, 2020): 如样本年龄集中和受教育程度偏高，与我国 劳动年龄人口占比提高、教育水平提升的现实情况 相符(王广州, 2019), 可见样本具有一定代表性。

\section{3 实证模型构建}

结构方程模型(Structural Equation Model, SEM) 是基于变量协方差矩阵来分析变量之间关系的一 种统计方法，即路径分析和因子分析的结合体(程 开明, 2006)。本研究涉及的态度、规范、知觉行为 
表1 本研究样本人口统计信息与人口普查和现有文献数据的比较

Table 1 Demographic characteristics of the samples in this study compared with the data of census and literatures

\begin{tabular}{|c|c|c|c|c|c|}
\hline $\begin{array}{l}\text { 统计变量 } \\
\text { Demographic va }\end{array}$ & riables & 频数 & 占比 & 2010年人口普查占比 & $\begin{array}{l}\text { 文献占比(Wang et al, 2020) } \\
\text { Percent in the literatures }\end{array}$ \\
\hline 性别 Gender & 男 Male & 503 & 52.9 & 51.1 & 46.45 \\
\hline & 女 Female & 447 & 47.1 & 48.9 & 53.55 \\
\hline 年龄 Age & 0-18岁 $0-18$ years old & 62 & 6.5 & 13.7 & 5.69 \\
\hline & 19-28岁 19-28 years old & 359 & 37.8 & 25.3 & 44.31 \\
\hline & 29-40岁 29-40 years old & 286 & 30.1 & 22.4 & 36.36 \\
\hline & 41-64岁 41-64 years old & 202 & 21.3 & 30.9 & 11.85 \\
\hline & $\geq 65$ 岁 Above 65 years old & 41 & 4.3 & 7.7 & 1.9 \\
\hline 受教育程度 & 小学及以下 Below primary school & 23 & 2.5 & 18.0 & 0.47 \\
\hline Education level & 初中 Middle school & 133 & 14.0 & 36.1 & 3.79 \\
\hline & 大专及高中 High school & 313 & 32.9 & 35.8 & 23.93 \\
\hline & 本科 Undergraduate & 366 & 38.5 & 9.1 & 60.19 \\
\hline & $\begin{array}{l}\text { 硕士及以上 Master and higher } \\
\text { academic degree }\end{array}$ & 104 & 12.1 & 1.0 & 10.66 \\
\hline 居住地区 & 东部地区 Eastern regions & 510 & 53.7 & 59.0 & - \\
\hline mesiderice & 中部地区 Central regions & 268 & 28.2 & 26.0 & - \\
\hline & 西部地区 Western regions & 172 & 18.1 & 15.0 & - \\
\hline
\end{tabular}

控制、保护意愿等潜变量均是不易直接测量的心理 学变量, 因而采用结构方程模型通过测量变量来 估计潜变量, 检验内生潜变量与外生潜变量之间 的关系, 并根据拟合优度指标验证模型结构的合 理性。

结构方程模型由测量模型和结构模型组成。测 量模型用于研究问卷题项、初阶因素、高阶因素之 间的关系，由以下方程决定：

$$
\begin{aligned}
& X=\Lambda_{X} \xi+\varepsilon \\
& Y=\Lambda_{Y} \eta+\delta
\end{aligned}
$$

其中, $\xi$ 为外生潜变量, 代表公众的非洲象保护态 度、规范、知觉行为控制、过去经验、其他个体特 征; $X$ 为外生潜变量对应的测量变量; $\eta$ 为内生潜变 量, 即保护意愿; $Y$ 为内生潜变量所对应的测量变量; $\Lambda_{X} 、 \Lambda_{Y}$ 为因素载荷系数矩阵; $\varepsilon 、 \delta$ 为测量误差。

结构方程用以研究潜变量之间的关系, 即外生 潜变量对内生潜变量的影响, 可以表示为:

$$
\eta=B \eta+\Gamma \xi+\zeta
$$

其中, $B$ 和 $\Gamma$ 代表方程的标准化回归系数矩阵, $\zeta$ 为无 法被解释的干扰向量。

本研究采用探索性结构方程建模(麦玉娇和温 忠麟, 2013): 首先评估量表的信度和效度, 具体来
说先进行克伦巴赫系数(Cronbach's $\alpha$ )、KMO值检验, 再结合探索性因子分析(Exploratory Factor Analysis, EFA) 解析问卷结构; 其次通过验证性因子分析 (Confirmatory Factor Analysis, CFA)检验测量模型 的合理性; 最后构建结构方程全模型进行模型拟合 检验及假设检验。由于样本数据量达到了题目的 10 倍或估计参数的 5 倍以上(Bentler \& Chou, 1987), 变 量均满足偏度小于 3 、峰度小于 8 , 近似服从正态分 布(吴明隆, 2010), 因此使用极大似然方法进行参数 估计。初阶验证性因子分析中, 变量之间具有高度 相关性, 考虑构建高阶模型 (邱皓政和林碧芳, 2009)。研究采用SPSS 21.0和Amos 21.0进行统计分 析和模型构建。

\section{3 实证分析结果}

\section{1 描述性统计结果}

问卷结果表明(附录3): (1) 68.5\%的受访者愿意 参与非洲象保护, 对于 “愿意参加非洲象保护活动” 的均值最高, 之后依次为 “将努力参加非洲象保护 活动”和“计划参加非洲象保护活动”。上述三个题项 平均值均高于中位数(4)。

(2)在态度方面, 公众行为态度(即认同保护活 动的重要性、必要性和趣味性)同意程度最高, 之后 
依次为对象态度(即认可非洲象的社会价值和生态 价值)和结果态度(即认同参与线上、线下及捐助保 护活动将对非洲象保护起到积极作用), 上述题项 平均值均高于 4 。关于非洲象经济价值的均值未达 到中位数(4), 公众回答存在明显差异, 标准差达到 2.338 。

(3)公众感知个体道德规范最强, 其次为命令 性规范和描述性规范。个人规范中, 均值从高到低 依次为 “参与非洲象保护符合道德原则” “有责任参 与非洲象保护活动” 和 “不参与非洲象保护活动会感 到内疚”; 命令性规范中, 感知来源从高到低依次 为国家法律、政府政策、朋友同事及同学、家人及 亲戚; 描述性规范中, 感知来源从高到低依次为政 府政策、社交媒体、一般媒体、朋友同事及同学、 家人及亲戚; 除朋友同事及同学、家人及亲戚的描 述性规范外, 上述题项平均值均高于中位数(4)。

(4)公众知觉行为控制题项均未达到中位数(4) 水平, 感知难度最大的是 “有足够的专业技能”, 之 后依次为“有足够经济能力”和“有足够时间”。

(5)仅 $30.1 \%$ 的受访者过去曾参与过其中某类活 动且很不频繁, $56.0 \%$ 参与过与其他野生动物保护 的相关活动且不太频繁。在知识方面, 42.1\%对非洲 象保护工作的相关组织、公益广告有一定了解, $22.2 \%$ 了解国内外的大象贸易管制规定，仅 $13.8 \% 了$ 解非洲象生存现状。受访者对参与渠道和参与方式 了解程度均值均未达到中位数(4)水平。

\section{2 信效度检验}

\subsection{1 量表信度和效度检验}

量表的可靠性和有效性可以通过信度和效度 来检验。首先, 采用克伦巴赫系数(Cronbach's $\alpha$ )作 为信度检验的标准, Cronbach's $\alpha>0.70$ 时具有高信 度, $0.35 \leq$ Cronbach's $\alpha<0.70$ 时具有一般信度。检 验结果表明, 量表总体的Cronbach's $\alpha$ 系数为 0.923 , 各潜变量的信度均满足标准(附录4), 量表可靠性较 高(Cronbach, 1951)。其次, 采用KMO检验和巴特利 特球形检验效度。 $\mathrm{KMO}$ 结果为 0.893 接近于 1 , 巴特 利特球形检验的 $\operatorname{sig}$ 值小于 0.001 , 可认为相关系数 矩阵具有显著差异, 适合做因子分析。最后, 基于 探索性因子分析解析量表的因子结构, 依据因子载 荷小于 0.5 或同时在两个主成分中载荷均大于 0.4 原 则剔除了4项观测题项, 最终保留29项观测题项, 提取4项公共因子, 对应于假设模型中态度(ATT)、
规范 $(N O R M)$ 、知觉行为控制 $(P B C)$ 、过去经验 $(P E)$ 等4项外生潜变量。

\subsection{2 测量模型信度与效度检验}

验证性因子分析用以检验测量模型的合理性。 由于“政府政策要求参与非洲象保护活动”和“国家 法律要求参与非洲象保护活动” 两观测题项的残差 之间存在共变关系, 剔除后避免了方法效应误差 (Landis, 2009)。模型结果显示, 标准化因子载荷均 大于 0.55 , 表明通过了项目检验, 具有良好的构念 效度(邱皓政和林碧芳, 2009)。各潜变量的组合信度 (CR)均大于 0.5 (附录 4 ), 表明模型测量稳定、可信度 高(Raines-Eudy, 2000); 平均变异萃取量(AVE)均大 于 0.5 , 表示潜变量被观测变量有效估计的聚玫程 度十分理想, 具有良好的操作性定义 (Landis, 2009)。所有潜在变量AVE的平方根均大于因素间的 相关系数(附录5), 代表潜变量具备理想的区分效度 (Qi \& Ploeger, 2018)。

\section{3 保护意愿模型拟合检验}

在结构方程模型中, 模型拟合度没有绝对的评 价标准, 一般通过观察多个指标进行综合评估(麦 玉娇和温忠麟, 2013)。本文选取了增值适配度指标、 绝对适配度指标和简约适配度指标三类九项评价 指标对模型拟合优度进行检验(温忠麟等, 2004; 邱 皓政和林碧芳, 2009; 吴明隆, 2010)。拟合检验结果 见表2, 所有检验结果均符合拟合标准, 表明结构 方程模型整体拟合效果良好。

\section{4 模型估计结果及假设检验}

假设检验结果见表 3 。态度、规范、知觉行为 控制、过去经验均对非洲象保护意愿具有显著正向 影响, 假设H1、H2、H3、H4得到验证。上述变量 中, 规范对保护意愿的影响最大(0.422), 其次是过 去经验 (0.253), 第三为知觉行为控制 $(0.160)$ 和态度 (0.156)。假设H5部分通过检验: 性别(-0.054)和西部 地区(0.066)虽然影响程度相对较小, 但通过了显著 性检验, 对保护意愿有显著影响, 即女性对非洲象 的保护意愿高于男性; 居住在西部地区的公众保 护意愿高于其他地区。东部居住区域对保护意愿影 响不显著, 仅存在微弱的正向影响。

图1显示了结构方程模型的路径系数。结果表 明: 公众非洲象保护态度包括保护对象态度、行为 态度、结果态度三个初阶因素, 解释能力从高到低 依次为行为态度 $(0.904)$ 、结果态度 $(0.803)$ 、对象态 
表2 我国公众非洲象保护意愿结构方程模型拟合检验结果

Table 2 Confirmatory analysis fitting indicators of the structural equation model to predict Chinese public willingness of African elephant conservation

\begin{tabular}{|c|c|c|c|c|c|c|c|c|c|}
\hline \multirow[t]{2}{*}{$\begin{array}{l}\text { 拟合指标 } \\
\text { Fit indices }\end{array}$} & \multicolumn{3}{|c|}{$\begin{array}{l}\text { 绝对适配度指标 } \\
\text { Absolute fit measurement }\end{array}$} & \multicolumn{3}{|c|}{$\begin{array}{l}\text { 增值适配度指标 } \\
\text { Incremental fit measurement }\end{array}$} & \multicolumn{3}{|c|}{$\begin{array}{l}\text { 简约适配度指标 } \\
\text { Parsimonious fit measurement }\end{array}$} \\
\hline & $\overline{\text { RMSEA }}$ & SRMR & GFI & $\overline{\text { TLI }}$ & CFI & NFI & $\overline{\mathrm{IFI}}$ & PNFI & $\chi^{2} / \mathrm{DF}$ \\
\hline 评价标准 Norms & $<0.08$ & $<0.08$ & $>0.90$ & $>0.90$ & $>0.90$ & $>0.90$ & $>0.90$ & $>0.50$ & $1<\chi^{2} / \mathrm{DF}<5$ \\
\hline 模型结果 Results & 0.050 & 0.060 & 0.920 & 0.929 & 0.938 & 0.914 & 0.938 & 0.799 & 3.373 \\
\hline
\end{tabular}

本文使用拟合检验指标估计模型拟合包括: 渐进残差均方和平方根(RMSEA)、标准化残差均方和平方根(SRMR)、适配度指标(GFI)、非规准适配指数 (TLI)、比较适配指数 $(\mathrm{CFI}) 、$ 规准适配指数(NFI)、增值适配指数(IFI)、简约调整后的规准适配指数( PNFI)、卡方自由度比 $\left(\chi^{2} / \mathrm{df}\right)$ 。

The overall model fit was estimated using statistical fit indices, Root Mean Square Error of Approximation (RMSEA), Standardized Root Mean Square Residual (SRMR), Goodness of Fit Index (GFI), Tucker-Lewis Fit Index (TLI), Comparative Fit Index (CFI), Normed Fit Index (NFI), Incremental Fit Index (IFI), Parsimony-adjusted Normed Fit Index (PNFI), and Chi-square per degree of freedom ratio ( $\left.\chi^{2} / \mathrm{df}\right)$.

表3 我国公众非洲象保护意愿结构方程模型假设检验结果

Table 3 Hypotheses testing results of the structural equation model to predict Chinese public willingness of African elephant conservation

\begin{tabular}{|c|c|c|c|c|c|}
\hline & $\begin{array}{l}\text { 估算系数 } \\
\text { Estimate }\end{array}$ & $\begin{array}{l}\text { 标准误 } \\
\text { S.E. }\end{array}$ & $\begin{array}{l}\text { 临界值 } \\
\text { C.R. }\end{array}$ & \multicolumn{2}{|c|}{$\begin{array}{l}\text { 假设检验结果 } \\
\text { Evaluation of hypotheses }\end{array}$} \\
\hline 态度 Attitude & $0.156^{* * *}$ & 0.083 & 3.914 & H1 & 接受 Supported \\
\hline 规范 Norm & $0.422^{* * *}$ & 0.058 & 7.889 & $\mathrm{H} 2$ & 接受 Supported \\
\hline 知觉行为控制 Perceived behavior control & $0.160^{* * *}$ & 0.036 & 4.218 & H3 & 接受 Supported \\
\hline 过去经验 Past experience & $0.253^{* * *}$ & 0.161 & 5.074 & $\mathrm{H} 4$ & 接受 Supported \\
\hline 性别 Gender & $-0.054^{*}$ & 0.059 & -2.258 & & \\
\hline 东部地区 Eastern regions & 0.058 & 0.080 & 1.858 & H5 & 部分接受 Partially supported \\
\hline 西部地区 Western regions & $0.066^{*}$ & 0.103 & 2.084 & & \\
\hline
\end{tabular}

$* * * P<0.001 ; * P<0.05$

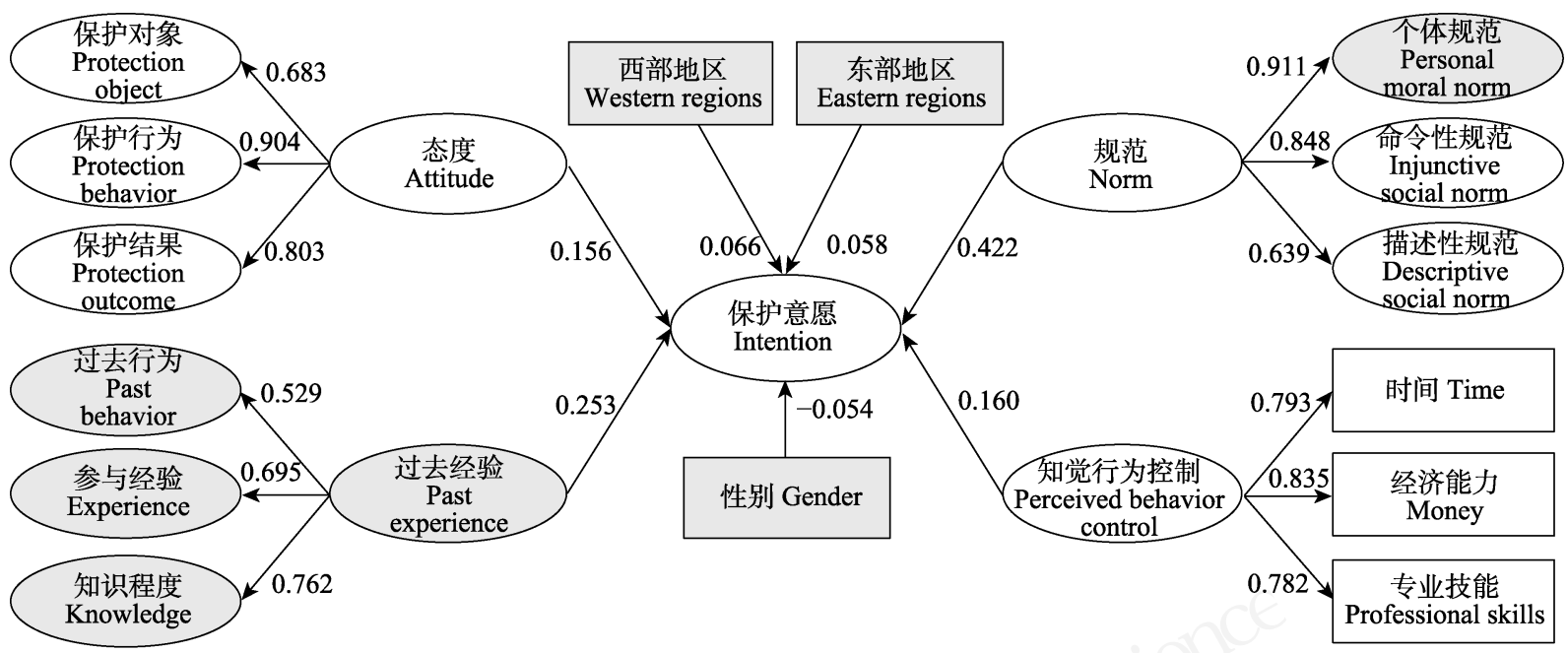

图1 我国公众的非洲象保护意愿结构方程模型路径图。灰色为拓展变量。

Fig. 1 Structural equation model, used to predict Chinese public willingness of African elephant conservation. Grey areas are expanded variable.

度(0.683)。规范包括个体规范、命令性规范、描述 性规范, 影响最大的是个体规范(0.911), 之后依次 是命令性规范(0.848)和描述性规范(0.639)。知觉行 为控制中经济能力解释力最强(0.835), 之后依次为
时间投入(0.793)和专业技能(0.782)。过去经验由过 去行为、参与经验、知识程度构成, 其中了解程度 及知识水平最为重要(0.762), 是否了解参与渠道和 参与方式次之(0.695), 最后为过去参与行为(0.529)。 


\section{4 结论与建议}

\section{1 结论}

总之, 我国公众对参与非洲象保护有积极性, 与Wang等(2018)、Xie (2020)的研究结果一致。然而, 公众保护意愿尚未达到强烈和十分强烈的程度, 在 非洲象保护中的积极作用仍有待进一步加强。基于 高阶结构方程模型结果, 本文得出以下结论:

(1)公众非洲象保护规范显著正向影响保护意 愿, 且是最重要的影响因素。与公众对国内野生动 物保护相比(Loyau \& Schmeller, 2017), 社会规范对 意愿的正向影响得到印证, 意味着政府宣传教育在 提升公众参与方面具有重要意义; 不同之处在于公 众参与国际物种保护方面, 规范的重要作用更加凸 显, 可能的原因一是拓展模型中纳入了个人规范, 增强了变量解释能力, 二是国内和国际物种保护工 作存在差异。

(2)过去经验显著正向影响保护意愿。其中, 知 识对意愿的积极影响与现有研究结果(周学红等, 2009)相似。针对国内物种保护, 学者更为关注受访 者观赏、接触野生动物的频率(Neupane et al, 2017), 而本研究有发现针对跨国物种保护意愿, 公众过去 参与保护行为和参与经验显著正向影响。为提升公 众国际野生动物保护意愿, 可从丰富参与经验、拓 宽参与渠道着手开展工作。

(3)知觉行为控制显著正向影响保护意愿。在国 内物种保护研究中, 知觉行为控制对物种接受度及 意愿影响不显著(Sakurai, 2014), 但对于跨境物种 保护, 公众的投入难度感知对保护参与意愿产生了 显著影响, 即公众在时间、经济、专业技能等的投 入方面感知难度越低, 保护意愿越强。

(4)保护态度显著正向影响保护意愿这一结论, 与现有研究关于态度对保护投入意愿、保护行为具 有积极影响的结果(Loyau \& Schmeller, 2017)一致。 公众保护对象态度、行为态度、结果态度越积极, 保 护意愿越强。

(5)性别负向影响保护意愿, 即女性可能具有 较强的保护意愿。与已有研究对比发现, 女性在国 内和国际物种保护中的意愿存在差异。BrowneNuñez等(2013)、Wilbur等(2018)研究发现女性对国 内野生动物带来的冲突和威胁感知更强烈, 保护意 愿较男性更低; 但本研究发现女性对跨境野生动物
有更强的保护意愿, 原因可能在于女性具有支持生 态保护的价值取向(Steger \& Witt, 1989), 对跨境物 种保护持更积极的态度(Wang et al, 2018)。

(6)从居住地区来看, 西部公众的保护意愿较 东部、中部略高, 与已有关于保护投入意愿的研究 结果不一致, Xie (2020)研究得出东部的公众对非洲 象保护投入意愿更强。究其原因, 一方面可能在于 从多维度度量与从保护投入意愿单维度度量导致 结果存在差异, 另一方面模型结果显示西部公众呈 现出更高的规范、知觉行为控制及过去经验。这意 味着东部地区尤其是过去象牙雕刻和加工较活跃 的城市, 保护政策宣传的实际成效不足。

\section{2 对策建议}

随着构建人类命运共同体的倡议得到越来越 多的国际认可, 发挥公众对非洲象等国际野生动物 保护的积极作用, 对于增强我国在推动建设人类共 同的生态安全美丽家园中的大国责任具有重要意 义。鉴于我国公众知觉行为控制较低、参与经验有 限、对非洲象保护理解不足等问题, 建议采取以下 措施提高公众保护意愿和引导公众积极参与。

(1)明确政策导向, 加强公众规范感知。管理部 门可从完善法律法规和理顺管理体制入手(刘鹏和 张伊静, 2020), 结合野生动物保护事业特点, 建立 健全社会公众参与机制, 严格执法、规范执法、做 好表率。各社会组织、媒体、基层社区积极配合, 营 造良好氛围, 提升公民参与国际野生动物保护的道 德义务感和社会责任感。

(2)加强宣传教育, 丰富公众保护知识并培育 积极态度。管理部门做好国际野生动物生存现状及 保护重要性的科学知识普及, 积极宣传非洲象等国 外物种的生存危机与保护不足(张丽荣等, 2020), 建 立“引导参与-工作落实-成效反馈”的良性循环机制, 增加与公众的互动交流(杜芳芝, 2017), 确保公众理 解保护的必要性、重要性及积极作用, 进而形成积 极的保护态度。

(3)拓宽参与渠道, 提高公众的知觉行为控制 能力。通过优化保护活动参与形式, 为公众参与创 造机会, 促使公众意愿落实到实际行动之中。一方 面借助现代化信息平台, 组织线上宣传、建立监督 渠道、开放募捐通道等, 降低公众对参与难度的预 期(王羽, 2020); 另一方面充分利用社会保护组织 资源, 面向野生动物保护工作者、志愿者开展专业 
技能培训, 提升参与能力。

(4)制定合理方案，提升地区保护活动成效。开 展针对性、精细化工作，调动不同主体参与积极性。 应特别关注位于东部过去象牙雕刻和加工比较活 跃的地区, 进一步完善保护工作机制、加强宣传教 育活动成效、形成积极参与的良好环境。

\section{ORCID}

张馨予 (D) https://orcid.org/0000-0002-6013-9974

\section{参考文献}

Ajzen I (1991) The theory of planned behavior. Organizational Behavior and Human Decision Processes, 50, 179-211.

Ajzen I (2011) The theory of planned behaviour: Reactions and reflections. Psychology \& Health, 26, 1113-1127.

Ajzen I (2019) Constructing a theory of planned behavior questionnaire. http://people.umass.edu/aizen/pdf/tpb.mea surement.pdf. (accessed on 2021-09-01)

Ajzen I, Fishbein M (2008) Scaling and testing multiplicative combinations in the expectancy-value model of attitudes. Journal of Applied Social Psychology, 38, 2222-2247.

Armitage CJ, Conner M (2001) Efficacy of the theory of planned behavior: A meta-analytic review. The British Journal of Social Psychology, 40, 471-499.

Bandara R, Tisdell C (2005) Changing abundance of elephants and willingness to pay for their conservation. Journal of Environmental Management, 76, 47-59.

Bentler PM, Chou CP (1987) Practical issues in structural modeling. Sociological Methods \& Research, 16, 78-117.

Brockington D, Scholfield K (2010) The conservationist mode of production and conservation NGOs in sub-Saharan Africa. Antipode, 42, 551-575.

Browne-Nuñez C, Jacobson SK, Vaske JJ (2013) Beliefs, attitudes, and intentions for allowing elephants in group ranches around Amboseli National Park, Kenya. Wildlife Society Bulletin, 37, 639-648.

Caughley G, Dublin H, Parker I (1990) Projected decline of the African elephant. Biodiversity Conservation, 54, 157-164.

Cheng KM (2006) Characteristics and application of structural equation model. Statistics and Decision, (10), 22-25. (in Chinese) [程开明 (2006) 结构方程模型的特点及应用. 统计与决策, (10), 22-25.]

Cialdini RB, Kallgren CA, Reno RR (1991) A focus theory of normative conduct, a theoretical refinement and reevaluation of the role of norms in human behavior. Advances in Experimental Social Psychology, 24, 201-234.

CITES (2019) Report on Monitoring the Illegal Killing of Elephants. https://cites.org/sites/default/files/eng/cop/18/doc/ E-CoP18-069-02-Add.pdf. (accessed on 2020-09-01)

Cronbach LJ (1951) Coefficient alpha and the internal structure of tests. Psychometrika, 16, 297-334.

Dalrymple CJ, Peterson MN, Cobb DT, Sills EO, Bondell HD, Dalrymple DJ (2012) Estimating public willingness to fund nongame conservation through state tax initiatives. Wildlife Society Bulletin, 36, 483-491.

Davies J, Foxall GR, Pallister J (2002) Beyond the intentionbehaviour mythology: An integrated model of recycling. Marketing Theory, 2, 29-113.

Dawes J (2008) Do data characteristics change according to the number of scale points used? An experiment using 5-point, 7-point and 10-point scales. International Journal of Market Research, 50, 61-104.

Du FZ (2017) Study on wild animal protection in public participation. Forest Investigation Design, (3), 70-71. (in Chinese with English abstract) [杜芳芝 (2017) 野生动物 保护中的公众参与研究. 林业勘查设计, (3), 70-71.]

Duan WT, Jiang GR (2008) A review of the theory of planned behavior. Advances in Psychological Science, 16, 315-320. (in Chinese with English abstract) [段文婷, 江光荣 (2008) 计划行为理论述评. 心理科学进展, 16, 315-320.]

EPI (2018) Stop Talking. Please Stop Talking. It is Now Time for Action. https://www.elephantprotectioninitiative.org/post/ inaugural-meeting-of-the-epi-consultative-group. (accessed on 2020-09-01)

Gray TNE, Gauntlett S (2017) Scale up elephant anti-poaching funds. Nature, 541, 157.

Haefele MA, Loomis JB, Lien AM, Dubovsky JA, Merideth RW, Bagstad KJ, Huang TK, Mattsson BJ, Semmens DJ, Thogmartin WE, Wiederholt R, Diffendorfer JE, LópezHoffman L (2019) Multi-country willingness to pay for transborder migratory species conservation: A case study of Northern Pintails. Ecological Economics, 157, 321-331.

Halkos GE, Jones N (2012) Modeling the effect of social factors on improving biodiversity protection. Ecological Economics, 78, 90-99.

Heiny J, Ajzen I, Leonhäuser IU, Schmidt P (2019) Intentions to enhance tourism in private households: Explanation and mediated effects of entrepreneurial experience. Journal of Entrepreneurship and Innovation in Emerging Economies, 5, 128-148.

Kideghesho JR (2016) Reversing the trend of wildlife crime in Tanzania: Challenges and opportunities. Biodiversity and Conservation, 25, 427-449.

Landis RS, Edwards BD, Cortina JM (2009) On the practice of allowing correlated residuals among indicators in structural equation models. In: Statistical and Methodological Myths and Urban Legends: Doctrine, Verity and Fable in the Organizational and Social Sciences (eds Lance CE, Vandenberg RJ), pp.193-215. Routledge, New York.

Langin C, Jacobson SK (2012) Risk and residency influences on public support for Florida panther recovery. Wildlife Society Bulletin, 36, 713-721.

Liu JZ (2020) Chinese plan for anti-poaching in Africa. Man 
and the Biosphere, (4), 67. (in Chinese) [刘建周 (2020) 为 非洲反盗猎提供“中国方案”. 人与生物圈, (4), 67.].

Liu P, Zhang YJ (2020) The regulation of wildlife trade in China: Current status, problems and policy suggestions. Nanjing Journal of Social Sciences, (5), 68-75. (in Chinese with English abstract) [刘鹏, 张伊静 (2020) 中国野生动 物市场监管: 现状、问题与优化对策. 南京社会科学, (5), 68-75.]

Loyau A, Schmeller DS (2017) Positive sentiment and knowledge increase tolerance towards conservation actions. Biodiversity and Conservation, 26, 461-478.

Mai YJ, Wen ZL (2013) Exploratory structural equation modeling (ESEM): An integration of EFA and CFA. Advances in Psychological Science, 21, 934-939. (in Chinese with English abstract) [麦玉娇, 温忠麟 (2013) 探 索性结构方程建模(ESEM): EFA和CFA的整合. 心理科 学进展, 21, 934-939.]

Martín-López B, Montes C, Benayas J (2007) The noneconomic motives behind the willingness to pay for biodiversity conservation. Biological Conservation, 139, 67-82.

Meng M, Xie Y (2013) Analysis of public participation in wild animal conservation. Chinese Journal of Wildlife, 34, 249-252. (in Chinese with English abstract) [梦梦, 谢屹 (2013) 浅析野生动物保护中的公众参与. 野生动物, 34, 249-252.]

Miller ZD (2017) The enduring use of the theory of planned behavior. Human Dimensions of Wildlife, 22, 583-590.

Morse-Jones S, Bateman IJ, Kontoleon A, Ferrini S, Burgess ND, Turner RK (2012) Stated preferences for tropical wildlife conservation amongst distant beneficiaries: Charisma, endemism, scope and substitution effects. Ecological Economics, 78, 9-18.

Neupane D, Kunwar S, Bohara AK, Risch TS, Johnson RL (2017) Willingness to pay for mitigating human-elephant conflict by residents of Nepal. Journal for Nature Conservation, 36, 65-76.

Ojea E, Loureiro ML (2007) Altruistic, egoistic and biospheric values in willingness to pay (WTP) for wildlife. Ecological Economics, 63, 807-814.

Phang SC, Failler P, Bridgewater P (2020) Addressing the implementation challenge of the global biodiversity framework. Biodiversity and Conservation, 29, 3061-3066.

Qi X, Ploeger A (2019) Explaining consumers' intentions towards purchasing green food in Qingdao, China: The amendment and extension of the theory of planned behavior. Appetite, 133, 414-422.

Qin TB, Yuan X (2021) China's practice to promote the biodiversity conservation in transboundary areas. Biodiversity Science, 29, 220-230. (in Chinese with English abstract) [秦天宝, 袁所 (2021) 推进生物多样性跨境区 域保护的中国实践. 生物多样性, 29, 220-230.]

Qiu HZ, Lin BF (2009) Principle and Application of Structural
Equation Model. China Light Industry Press, Beijing. (in Chinese) [邱皓政, 林碧芳 (2009) 结构方程模型的原理 与应用. 中国轻工业出版社, 北京.]

Raines-Eudy R (2000) Using structural equation modeling to test for differential reliability and validity: An empirical demonstration. Structural Equation Modeling: A Multidisciplinary Journal, 7, 124-141.

Ru XJ, Qin HB, Wang SY (2019) Young people's behaviour intentions towards reducing PM2.5 in China: Extending the theory of planned behaviour. Resources, Conservation and Recycling, 141, 99-108.

Sakurai R, Enari H, Matsuda T (2014) Testing socialpsychological theories to predict residents' behavioral intentions regarding wildlife issues. Honyurui Kagaku, 54, 219-230.

Schwartz SH (1977) Normative influences on altruism. Advances in Experimental Social Psychology, 10, 221-279.

Shi HT, Wang ZY, Yan L (2019) The influence of ecological cognition on farmers' grain for green behavior: Based on theory of planned behavior and multi-group SEM. China Land Science, (3), 42-49. (in Chinese with English abstract) [史恒通, 王铮钰, 阎亮 (2019) 生态认知对农户退耕还 林行为的影响——基于计划行为理论与多群组结构方程 模型. 中国土地科学, (3), 42-49.]

Shi XY, Zhang XC, Xiao LY, Li BB, Liu JM, Yang FY, Zhao X, Cheng C, Lv Z (2020) Public perception of wildlife consumption and trade during the COVID-19 outbreak. Biodiversity Science, 28, 630-643. (in Chinese with English abstract) [史湘芗, 张晓川, 肖凌云, 李涁涁, 刘金梅, 杨 方义, 赵翔, 程琛, 吕植 (2020) 新冠肺炎时期公众对野 生动物消费和贸易意愿的调查. 生物多样性, 28 , 630-643.]

Steger MAE, Witt SL (1989) Gender differences in environmental orientations: A comparison of publics and activists in Canada and the U. S. Political Research Quarterly, 42, 627-649.

Subroy V, Rogers AA, Kragt ME (2018) To bait or not to bait: A discrete choice experiment on public preferences for native wildlife and conservation management in western Australia. Ecological Economics, 147, 114-122.

Tonglet M, Phillips PS, Read AD (2004) Using the theory of planned behaviour to investigate the determinants of recycling behavior: A case study from Brixworth, UK. Resources, Conservation and Recycling, 41, 191-214.

Turpie JK (2003) The existence value of biodiversity in South Africa: How interest, experience, knowledge, income and perceived level of threat influence local willingness to pay. Ecological Economics, 46, 199-216.

Wang C (2020) Research on the external communication of international NGOs. Journalism Communication, (18), 105-106. (in Chinese) [王羽 (2020) 国际非政府组织对外 传播研究一以美国非政府组织“野生救援(WILDAID)” 为例. 新闻传播, (18), 105-106.] 
Wang GZ (2019) 70 years of China: The changes of population age structure and the trend of population aging. Chinese Journal of Population Science, (3), 2-15, 126. (in Chinese with English abstract) [王广州 (2019) 新中国70年: 人口 年龄结构变化与老龄化发展趋势. 中国人口科学, (3), 2-15, 126.]

Wang SK, Cai Z, Hu YX, Cirella GT, Xie Y (2020) Chinese resident preferences for African elephant conservation: Choice experiment. Diversity, 12, 453.

Wang ZZ, Gong YZ, Mao XQ (2018) Exploring the value of overseas biodiversity to Chinese netizens based on willingness to pay for the African elephants' protection. Science of the Total Environment, 637-638, 600-608.

Wen ZL, Hou JT, Marsh HW (2004) Structural equation model testing: Cutoff criteria for goodness of fit indices and chi-square test. Acta Psychologica Sinica, 36, 186-194. (in Chinese with English abstract) [温忠麟, 侯杰泰, 马什赫伯 特 (2004) 结构方程模型检验: 拟合指数与卡方准则. 心 理学报, 36, 186-194.]

Wilbur RC, Lischka SA, Young JR, Johnson HE (2018) Experience, attitudes, and demographic factors influence the probability of reporting human-black bear interactions. Wildlife Society Bulletin, 42, 22-31.

Wu ML (2010) Structural Equation Modeling: Operation and Application of AMOS. Chongqing University Press, Chongqing. (in Chinese) [吴明隆 (2010) 结构方程模型: AMOS的操作与应用. 重庆大学出版社, 重庆.]

Xie Y (2020) Ecological labeling and wildlife conservation: Citizens' perceptions of the elephant ivory-labeling system in China. Science of the Total Environment, 702, 134709.

Yuan DD (2018) Global ivory trade and elephant protection. Ecological Economy, 34(3), 2-5. (in Chinese) [袁丹丹 (2018) 全球象牙贸易与大象保护. 生态经济, 34(3), 2-5.]

Zhang LR, Meng R, Jin SC, Pan Z, Zhou J, Dong JC, Wang XH, Wang JN, Chang JW (2020) Protecting wildlife by the strictest instruments: China's current situation and reform direction. Chinese Journal of Environmental Management, (2), 5-19. (in Chinese with English abstract) [张丽荣, 孟锐, 金世超, 潘哲, 周佳, 董金池, 王夏晖, 王金南, 常纪文 (2020) 实施最严格的野生动物保护: 中国现状与改革方 向. 中国环境管理, (2), 5-19.]

Zhou XH, Ma JZ, Zhang W, Wang Q (2009) Evaluating the economic value and its reliability analysis of endangered species conservation with contigent method-A case study on the willingness to pay of the citizens in Harbin on Amur. Journal of Natural Resources, 24, 276-285. (in Chinese with English abstract) [周学红, 马建章, 张伟, 王强 (2009) 运 用CVM评估濒危物种保护的经济价值及其可靠性分析 一以哈尔滨市区居民对东北虎保护的支付意愿为例. 自然资源学报, 24, 276-285.]

Zhou XH, Wang Q, Zhang W, Jin Y, Wang Z, Chai Z, Zhou ZQ, Cui XF, MacMillan DC (2018) Elephant poaching and the ivory trade: The impact of demand reduction and enforcement efforts by China from 2005-2017. Global Ecology \& Conservation, 16, e00486.

(责任编委: 李涁涁 责任编辑: 时意专)

\section{附录 Supplementary Material}

附录1 公众非洲象保护意愿影响因素分析模型(修改自Ajzen, 1991)

Appendix 1 Conceptual model of public protection intention of African elephant (adapted from Ajzen, 1991)

https://www.biodiversity-science.net/fileup/PDF/2021082-1.pdf

附录2 中国公众关于非洲象保护意愿的研究问卷

Appendix 2 Questionnaire on Chinese public willingness of African elephant protection

https://www.biodiversity-science.net/fileup/PDF/2021082-2.pdf

附录3 公众非洲象保护意愿及影响因素的测量题项描述性统计分析

Appendix 3 Descriptive statistics of key variable indicators of public protection intention of African elephant https://www.biodiversity-science.net/fileup/PDF/2021082-3.pdf

附录4 结果方程模型信度和聚敛效度检验结果

Appendix 4 The results of reliability and average variance extracted https://www.biodiversity-science.net/fileup/PDF/2021082-4.pdf

附录5 结构方程模型区别效度检验

Appendix 5 The results of discriminant validity

https://www.biodiversity-science.net/fileup/PDF/2021082-5.pdf 
张馨予, 胡宇轩, 张忠义, 傅铎涵, 谢屹 (2021) 中国公众的国际野生动物保护意愿调查: 以非洲象为例. 生物多样性, 29, 1358-1368. http://www.biodiversity-science.net/CN/10.17520/biods.2021082

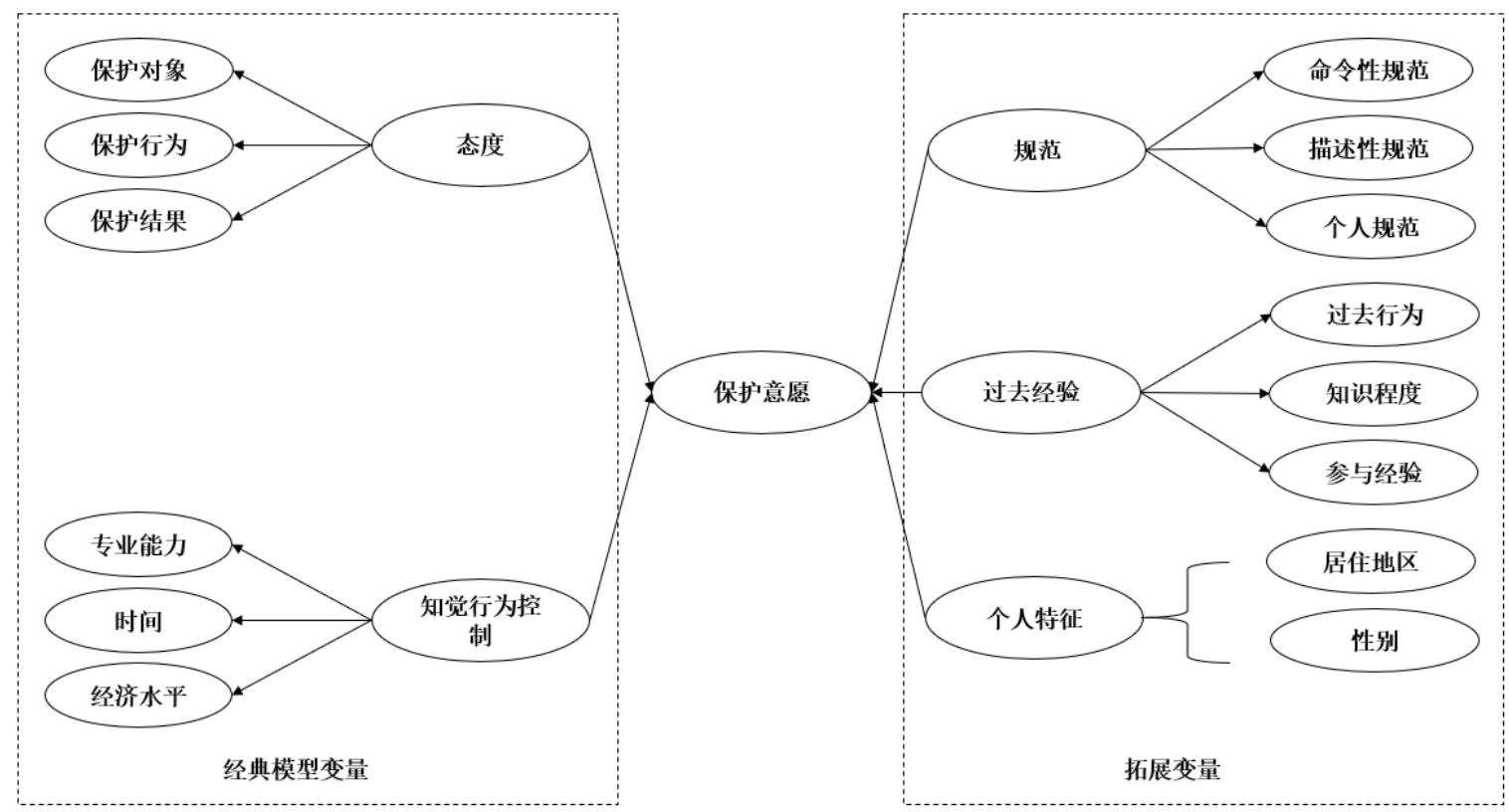

附录1 公众非洲象保护意愿影响因素分析模型 (修改自Ajzen, 1991)

Appendix 1 Conceptual model of public protection intention of African elephant (adapted from Ajzen, 1991) 
张馨予, 胡宇轩, 张忠义, 傅钰涵, 谢屹 (2021) 中国公众的国际野生动物保护意愿调查: 以非洲象为例. 生物多样性, 29, 1358-1368

http://www.biodiversity-science.net/CN/10.17520/biods.2021082

附录2 我国公众关于非洲象保护意愿的研究问卷

Appendix 2 Questionnaire on Chinese public willingness of African elephant protection

1. 请勾选出您对以下活动的参与程度。

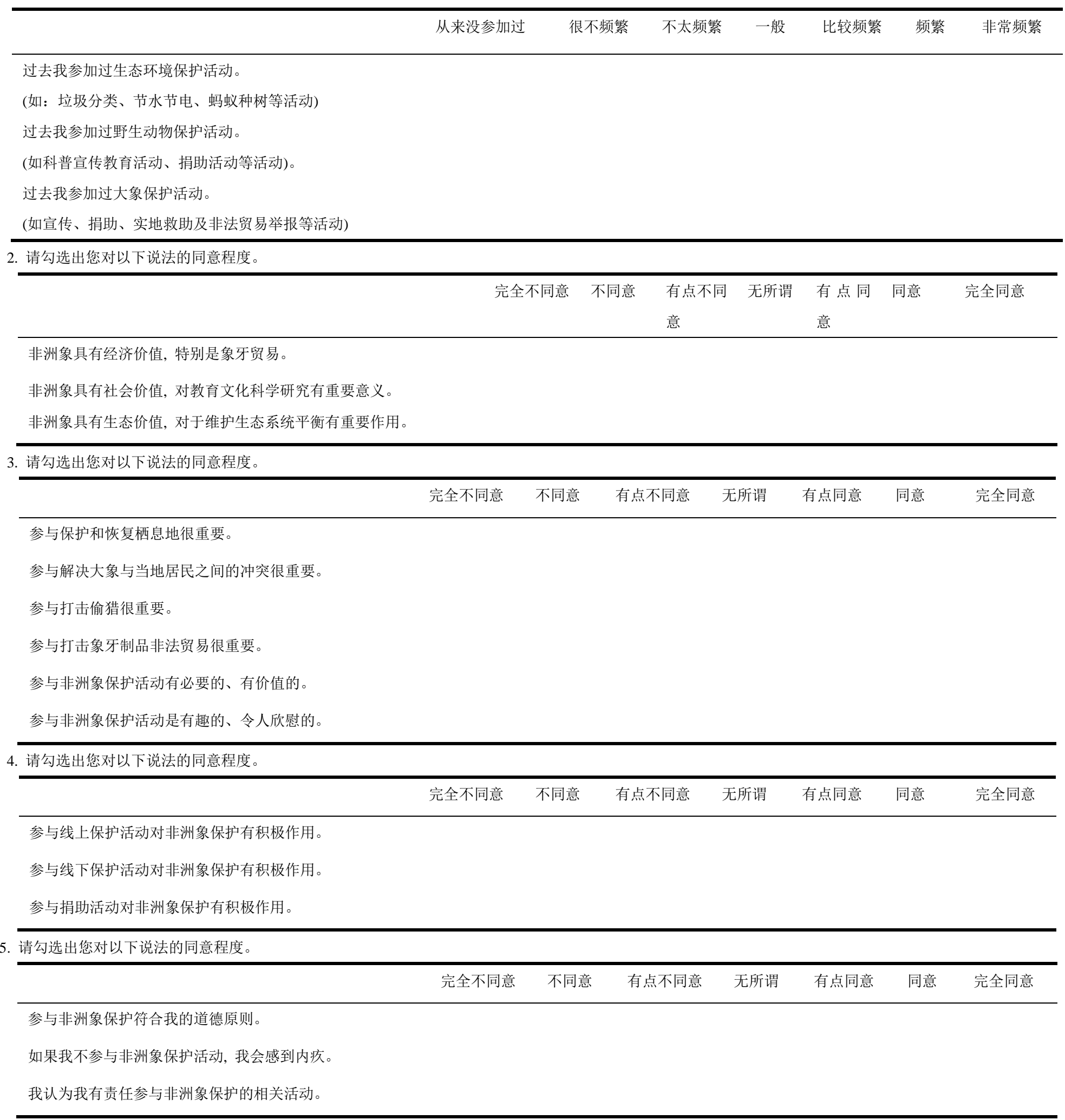

6. 请勾选出您对以下说法的同意程度。

\begin{tabular}{|c|c|c|c|c|c|c|}
\hline $\begin{array}{l}\text { 完全不同 } \\
\text { 意 }\end{array}$ & 不同意 & $\begin{array}{l}\text { 有点不同 } \\
\text { 意 }\end{array}$ & 无所谓 & $\begin{array}{l}\text { 有 点 } \\
\text { 同意 }\end{array}$ & 同意 & $\begin{array}{l}\text { 完全同 } \\
\text { 意 }\end{array}$ \\
\hline
\end{tabular}

我的家人或亲戚都认为我应该参与非洲象保护活动。

我的朋友、同事及同学都认为我应该我参与非洲象保护活动。

政府出台的政策认为我应该参与非洲象保护活动。

国家制定的法律认为我应该参与非洲象保护活动。

7. 请勾选出您对以下说法的同意程度。

\begin{tabular}{|c|c|c|c|c|c|c|}
\hline 完 全 不 & 不同意 & 有点不 & 不清楚 & 有点同 & 同意 & 完全同 \\
\hline 同意 & & 同意 & & 意 & & 意 \\
\hline
\end{tabular}


张馨予, 胡宇轩, 张忠义, 傅钰涵, 谢屹 (2021) 中国公众的国际野生动物保护意愿调查: 以非洲象为例. 生物多样性, 29, 1358-1368. http://www.biodiversity-science.net/CN/10.17520/biods.2021082

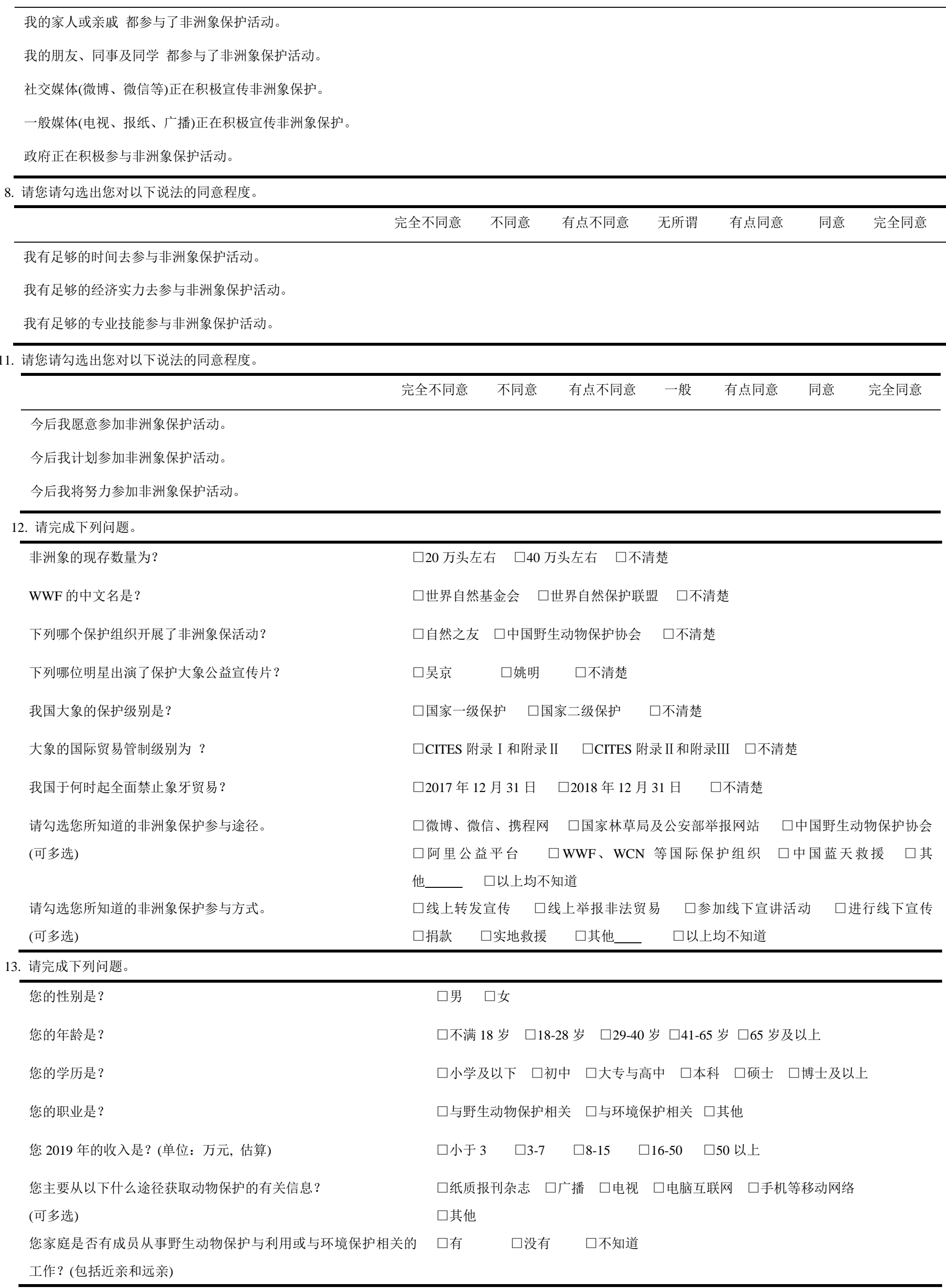


张馨予, 胡宇轩, 张忠义, 傅钰涵, 谢屹 (2021) 中国公众的国际野生动物保护意愿调查: 以非洲象为例. 生物多样性, 29, 1358-

1368 .

http://www.biodiversity-science.net/CN/10.17520/biods.2021082

附录3 公众非洲象保护意愿及影响因素的测量题项描述性统计分析

Appendix 3 Descriptive statistics of key variable indicators of public protection intention of African elephant

\begin{tabular}{|c|c|c|c|c|}
\hline 初阶因子 & 序号 & 测量题项 & 平均值 & 标准差 \\
\hline Primary factor & Number & Observation variable & Mean & Standard deviation \\
\hline \multirow[t]{3}{*}{ 保护意愿 } & $\mathrm{I} 1$ & 愿意参加非洲象保护活动 & 5.201 & 1.524 \\
\hline & I 2 & 计划参加非洲象保护活动 & 4.540 & 1.564 \\
\hline & $\mathrm{I} 3$ & 将努力参加非洲象保护活动 & 4.747 & 1.601 \\
\hline \multirow[t]{3}{*}{ 保护对象态度 } & A1 & 非洲象具有经济价值 & 3.593 & 2.338 \\
\hline & $\mathrm{A} 2$ & 非洲象具有社会价值 & 5.821 & 1.354 \\
\hline & $\mathrm{A} 3$ & 非洲象具有生态价值 & 6.353 & 1.056 \\
\hline \multirow[t]{3}{*}{ 保护行为态度 } & A4 & 参与非洲象保护活动重要 & 6.257 & 0.882 \\
\hline & A5 & 参与非洲象保护活动必要 & 6.402 & 0.900 \\
\hline & A6 & 参与非洲象保护活动有趣 & 5.954 & 1.235 \\
\hline \multirow[t]{3}{*}{ 保护结果态度 } & A7 & 线上保护活动对非洲象保护有积极作用 & 5.722 & 1.337 \\
\hline & A8 & 线下保护活动对非洲象保护有积极作用 & 6.196 & 1.066 \\
\hline & A9 & 捐助活动对非洲象保护有积极作用 & 5.834 & 1.264 \\
\hline \multirow[t]{3}{*}{ 个体规范 } & N1 & 不参与非洲象保护活动会感到内疚 & 4.482 & 1.585 \\
\hline & $\mathrm{N} 2$ & 有责任参与非洲象保护活动 & 5.035 & 1.511 \\
\hline & $\mathrm{N} 3$ & 参与非洲象保护符合道德原则 & 6.066 & 1.166 \\
\hline \multirow[t]{4}{*}{ 命令性规范 } & N4 & 家人或亲戚认为应该参与非洲象保护活动 & 4.300 & 1.694 \\
\hline & N5 & 朋友、同事及同学认为应该参与非洲象保护活动 & 4.387 & 1.642 \\
\hline & N6 & 政府政策要求参与非洲象保护活动 & 4.800 & 1.582 \\
\hline & N7 & 国家法律要求参与非洲象保护活动 & 4.825 & 1.639 \\
\hline \multirow[t]{5}{*}{ 描述性规范 } & N8 & 家人或亲戚正在积极参与非洲象保护 & 3.287 & 1.794 \\
\hline & N9 & 朋友、同事及同学正在积极参与非洲象保护 & 3.496 & 1.726 \\
\hline & N10 & 社交媒体如微博、微信等正在积极宣传非洲象保护 & 4.720 & 1.618 \\
\hline & N11 & 一般媒体如电视、报纸等正在积极宣传非洲象保护 & 4.705 & 1.585 \\
\hline & N12 & 政府正在积极参与非洲象保护活动 & 4.798 & 1.626 \\
\hline \multirow[t]{3}{*}{ 知觉行为控制 } & P1 & 有足够的时间参与非洲象保护活动 & 3.399 & 1.754 \\
\hline & $\mathrm{P} 2$ & 有足够的经济能力参与非洲象保护活动 & 3.094 & 1.681 \\
\hline & $\mathrm{P} 3$ & 有足够的专业技能参与非洲象保护活动 & 2.752 & 1.678 \\
\hline \multirow[t]{2}{*}{ 过去行为 } & B1 & 过去参加过大象保护活动 & 1.606 & 1.121 \\
\hline & B2 & 过去参加过其他野生动物保护活动 & 2.199 & 1.376 \\
\hline \multirow[t]{2}{*}{ 参与经验 } & B3 & 非洲象保护参与渠道的了解程度 & 2.083 & 1.794 \\
\hline & B4 & 非洲象保护参与方式的了解程度 & 3.149 & 2.021 \\
\hline \multirow[t]{2}{*}{ 知识程度 } & B5 & 非洲象生存现状了解程度 & 1.355 & 1.977 \\
\hline & B6 & 非洲象保护工作开展现状了解程度 & 2.946 & 2.102 \\
\hline
\end{tabular}

现有参与渠道包括微博、微信及携程等网络平台、国家林草局及公安部举报网站、阿里公益平台、中国野生动物保护 协会、中国蓝天救援等国内民间组织及世界自然基金会等国际保护组织开展的非洲象保护活动。参与方式包括线上转 发公益广告和报道、监督和报告在线非法交易，以及线下活动，诸如宣传教育、实地救助和捐助活动等。 
张馨予, 胡宇轩, 张忠义, 傅钰涵, 谢屹 (2021) 中国公众的国际野生动物保护意愿调查: 以非洲象为例. 生物多样性, 29, 13581368.

http://www.biodiversity-science.net/CN/10.17520/biods.2021082

附录4 结果方程模型信度和聚敛效度检验结果

Appendix 4 The results of reliability and average variance extracted

\begin{tabular}{llll}
\hline & Cronbach's Alpha & Composite reliability & Average variance extracted \\
\hline 态度 Attitude & 0.832 & 0.924 & 0.576 \\
规范 Norm & 0.922 & 0.962 & 0.782 \\
知觉行为控制 & 0.843 & 0.846 & 0.646 \\
Perceived behavior control & & & \\
过去经验 Past experience & 0.695 & 0.874 & 0.540 \\
保护意愿 Intention & 0.909 & 0.910 & 0.771 \\
\hline
\end{tabular}


张馨予, 胡宇轩, 张忠义, 傅钰涵, 谢屹 (2021) 中国公众的国际野生动物保护意愿调查: 以非洲象为例. 生物多样性, 29, 13581368.

http://www.biodiversity-science.net/CN/10.17520/biods.2021082

附录5 结构方程模型区别效度检验

Appendix 5 The results of discriminant validity

\begin{tabular}{|c|c|c|c|c|c|}
\hline & 保护意愿 & 态度 & 规范 & 知觉行为控制 & 过去经验 \\
\hline & Intention & Attitude & Norm & Perceived behavior control & Past experience \\
\hline 保护意愿 Intention & 0.878 & & & & \\
\hline 态度 Attitude & $0.499^{* * *}$ & 0.759 & & & \\
\hline 规范 Norm & $0.735^{* * *}$ & $0.561^{* * *}$ & 0.884 & & \\
\hline 知觉行为控制 & $0.527^{* * *}$ & $0.149^{* * *}$ & $0.573^{* * *}$ & 0.804 & \\
\hline \multicolumn{6}{|l|}{ Perceived behavior control } \\
\hline 过去经验 Past experience & $0.579^{* * *}$ & $0.304^{* * *}$ & $0.495^{* * *}$ & $0.386^{* * *}$ & 0.735 \\
\hline
\end{tabular}

对角线指AVE的平方根, 对角线下方为相关系数; ${ }^{* * *}$ 表示在 $1 \%$ 水平上显著。

The diagonal values represent the square root of AVE and the values below the diagonal represent the correlation coefficients. ${ }^{* * *}$ The correlation is signifcant at the 0.001 level. 